\title{
Isobar separation and precision mass spectrometry of short-lived nuclides with a multi-reflection time-of-flight analyzer
}

\author{
Lutz Schweikhard ${ }^{1}$, Marco Rosenbusch, Frank Wienholtz, Robert N. Wolf ${ }^{2}$ \\ Ernst-Moritz-Arndt-Universität \\ Institute of Physics \\ Felix-Hausdorff-Str. 6 \\ 17489 Greifswald, Germany \\ E-mail: Ischweik@uni-greifswald.de
}

\begin{abstract}
Multi-Reflection Time-of-Flight Mass Spectrometry (MR-ToF MS) is a technique that has recently been introduced to nuclear physics research. The method is reviewed and several examples from ISOLTRAP at ISOLDE/CERN are given that illustrate its power and usefulness. Initially built for fast separation of the ions of interest from abundant contaminations in order to allow precision mass spectrometry, which was demonstrated in the case of ${ }^{82} \mathrm{Zn}$, ISOLTRAP's multi-reflection time-of-flight mass spectrometer also succeeded in measuring the previously unknown mass values of ${ }^{53} \mathrm{Ca}$ and ${ }^{54} \mathrm{Ca}$. In addition, proof-of-principle experiments have been performed which demonstrate further applications where fast and high-resolution separation of ions of interest from mixtures with high abundances of contamination species is required. Furthermore, in an off-line setup MR-ToF MS is explored with respect to space-charge effects in order to find the limits of the ion abundances that can be handled. The phenomena observed include peak coalescence in the time-of-flight spectra and different distributions of the kinetic energies of the stored ion species.
\end{abstract}

$X$ Latin American Symposium on Nuclear Physics and Applications (X LASNPA)

1-6 December 2013

Montevideo, Uruguay

\footnotetext{
${ }^{1}$ Speaker

2 present address of R.N. Wolf: Max-Planck-Institut für Kernphysik, Saupfercheckweg 1, D-69117 Heidelberg, Germany
} 


\section{Introduction}

The mass is one of the most fundamental properties of atomic nuclei as it is directly connected to the binding energies of its nucleons and thus with the structure of the nuclei. Already a century ago mass spectrometry had significant input in nuclear physics when new isotopes and the "mass defect" were discovered. Today, precision mass spectrometry reaches out to more and more exotic nuclides [1-3]. While storage-ring based mass spectrometry at high kinetic energies gives access to nuclides with half-lives down to the microsecond range [4], state-of-the-art studies of longer-lived species (still down to a few tens of milliseconds) are mostly performed by Penning-trap mass spectrometry. Cyclotron-frequency measurements of exotic ions stored in high magnetic fields were introduced almost three decades ago by the ISOLTRAP experiment [5] and are now used at several facilities around the world [2].

Due to the production and ionization processes of exotic nuclides, a major obstacle in precision Penning-trap mass spectrometry is the "contamination" with long-lived and stable species, as the Coulomb interaction leads to cyclotron-frequency shifts which can exceed the intended precision by orders of magnitude [6,7]. Besides performing mass measurements, the Penning trap can also be used for mass purification [8] and thus contamination suppression for ions with typical half-lives of several $100 \mathrm{~ms}$ and above. However, as more challenging scenarios are addressed, this method reaches its limits with respect to the ions' intensity ratios [9] and the required duration of ion separation. In order to speed up and to enhance the purification, isobaric mass separation by use of a multi-reflection time-of-flight (MR-ToF) mass analyzer [10] has recently been added to the experimental event sequence [11]. In addition, this device can be used as a mass spectrometer of its own [12-14]. It allows precision measurements as well as ion-intensity monitoring at high, i.e. isobar-selective, resolving power, which is useful for many studies beyond precision mass determination $[15,16]$.

In this contribution, the basics of time-of-flight mass spectrometry related to the multireflection method are shortly reviewed as well as the ISOLTRAP setup and the achievements of its MR-ToF mass analyzer. In addition, recent preliminary results from an off-line setup are reported, where the properties and limits of MR-ToF mass spectrometry with respect to ion-ion interactions and space-charge effects are investigated.

\section{Time-of-Flight Mass Spectrometry}

Time-of-flight mass spectrometry is based on the mass-over-charge dependent time ions travel on a well-defined path from a source to a detector. If the ions have identical kinetic energies, start positions and times, their flight times between the source ("S", Fig. 1a) and the detector ("D") are proportional to the square root of their mass-over-charge ratios. The energy is given by starting in an acceleration section with a homogenous electric field. In general, different initial positions in the source lead to different flight times. But by use of two acceleration sections of appropriate field strengths [17], a first-order focusing, i.e. minimization of the width of the arrival-time distribution, can be achieved for arbitrary detector positions. 
However, this width is also affected by the ions' energy distribution prior to their acceleration. In particular, for a thermal distribution, there are ions with initial velocities in the direction towards the detector and others with opposite direction. The latter, which travel against the electric force, are slowed down, stopped and accelerated back to their initial position where they continue just like their counterparts that started in the "forward direction" right from the beginning. The time difference associated with this "turnaround" cannot be compensated but limits the resolving power. In order to keep it low, the accelerating electric field has to be high. But a high field leads to a correspondingly high position-related energy distribution. The invention of the reflectron [18] was a major step to minimize the flight-time consequences of this effect: Ions with higher energies penetrate deeper into an ion-optical mirror that reflects them towards the detector (Fig. 1b). The longer ion trajectory compensates the higher energy and, again in first order, ions of different initial energy have the same total flight time.

(a)

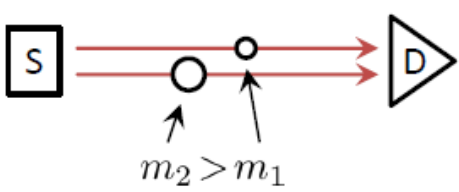

(b)

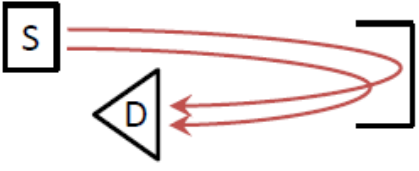

(c)

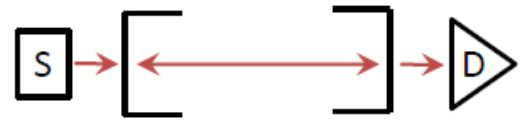

(d)

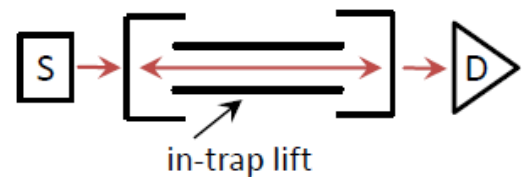

Fig. 1: Sketches of time-of-flight mass spectrometry devices. For details see text.

The initial temporal ion-pulse width, i.e. the remaining turnaround time and possibly a finite duration of ion creation in the source (or of the injection into the acceleration zone), limits the temporal distribution $\Delta t$ of the total flight time $t$ and thus the resolving power, $m / \Delta m=t / 2 \Delta t$. To increase $m / \Delta m$, the flight time has to be extended, either via very long drift sections or by use of a second ion mirror to fold the ion path several tens, hundreds or thousands of times by multiple reflections [10] (Fig. 1c). In this arrangement the ions pass the first mirror ("injection"), are trapped between the mirrors, and are finally released by passing the second mirror ("ejection") towards the ion detector. Thus, this multi-reflection device can not only be used as a time-of-flight mass spectrometer, but also as an ion storage device [19-21], in which case it is often referred to as "electrostatic ion-beam trap" or similar.

\section{MR-ToF studies at ISOLTRAP}

A few MR-ToF mass analyzers have already been installed at radioactive-beam facilities and further setups are currently under construction or planned, as the advantages with respect to accessibility of nuclides with very short half-lives and/or low production yields are obvious. Offline experiments have been performed at Oak Ridge [22] and spectra of short-lived nuclides (with already known mass values) were taken at RIKEN [13] and at GSI [14]. Beyond these 
proof-of-principle studies, the ISOLTRAP collaboration recently reported the first use of an MR-ToF device for mass separation followed by a Penning-trap measurement of a previously unknown mass value [23] as well as the first determination of so far unmeasured mass values by use of its MR-ToF component as a mass spectrometer of its own [12]. As also demonstrated at ISOLTRAP [7,24], several other research areas can profit from the MR-ToF device (see section 3.3). In the following the properties of ISOLTRAP and its MR-ToF device are summarized.

\subsection{Overview of the ISOLTRAP experiment}

ISOLTRAP was the first Penning-trap mass spectrometer for short-lived nuclides [5,25]. It was installed at ISOLDE/CERN in the 1980s and continuously further developed over time [24,26,27]. Currently it includes four ion-trapping devices as sketched in Fig. 2.
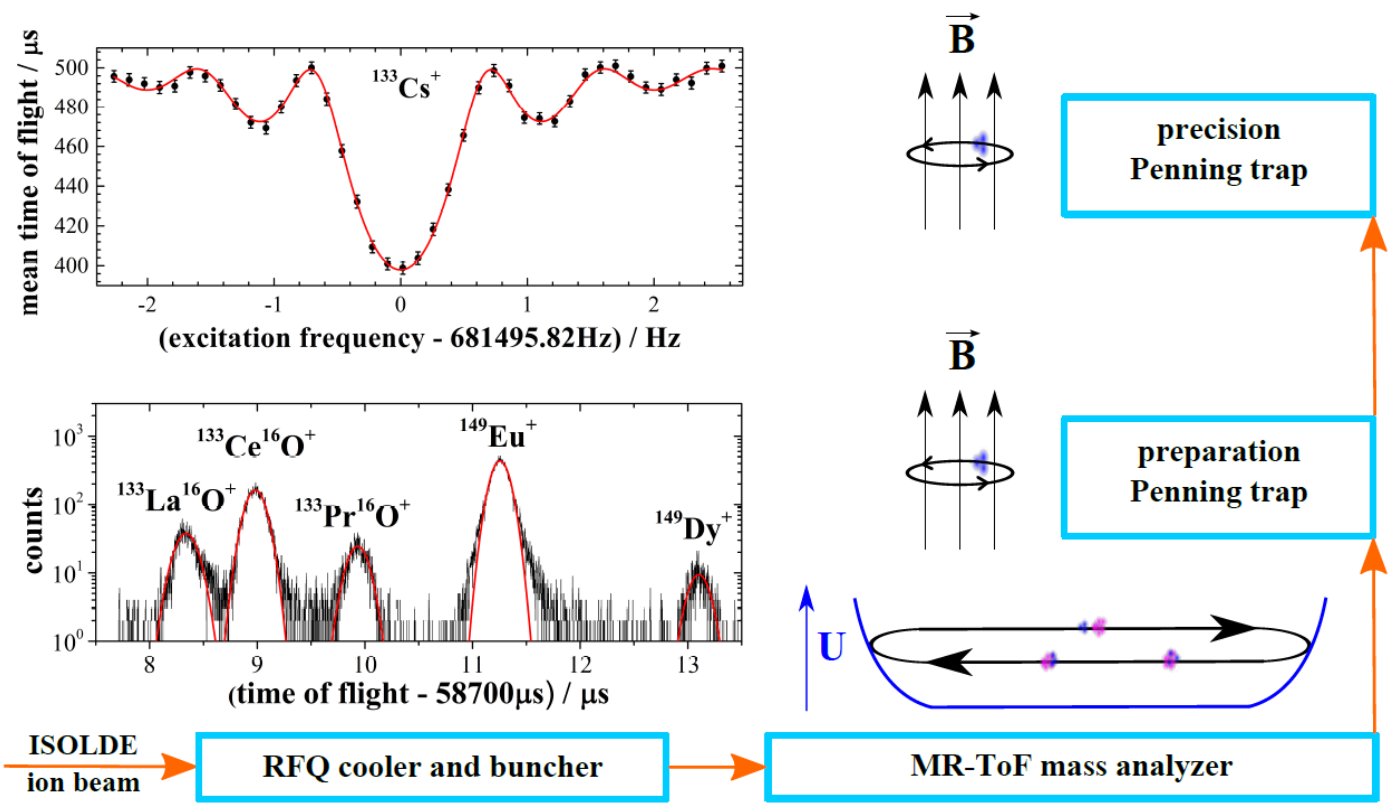

Fig. 2: Major components of ISOLTRAP and typical mass spectra. For details see text.

Exotic ions are delivered from the online mass separator ISOLDE [28] as quasi-continuous beams with up to $60 \mathrm{keV}$ kinetic energy (for singly charged ions). Typically, the ions are decelerated, accumulated, bunched, and cooled in a linear radio-frequency quadrupole (RFQ) Paul trap [29], transferred to the MR-ToF component for a first mass selection, then send to a first Penning trap [30] for further mass selection (if necessary) and for preparation [31], and finally forwarded to a second Penning trap for time-of-flight ion-cyclotron-resonance (ToFICR) mass measurements [32,33] with relative uncertainties down to $8 \times 10^{-9}$ [34].

Before the installation of the MR-ToF device, the ions were transferred directly from the Paul trap to the preparation Penning trap. It is still possible to let the ions pass though the MRToF section without multiple reflections. On the other hand, mass measurements can also be performed by the MR-ToF component, i.e. without forwarding the ions to the Penning traps. 


\subsection{The MR-ToF mass analyzer at ISOLTRAP}

ISOLTRAP's MR-ToF device has been built at the University of Greifswald [35] based on the design of the electrostatic ion-beam trap of Zajfman and co-workers at the Weizmann Institute of Science, Rehovot [19,36]. It has a length of about $80 \mathrm{~cm}$ and consists of two mirrors each composed of six electrodes and a 46-cm long center electrode which acts as an "in-trap lift" [37]: Generally, in other MR-ToF devices, the mirror potentials are lowered for injection and ejection. However, these potentials should be as stable as possible and, thus, switching of the mirror voltages should be avoided. This can be achieved by use of the in-trap potential lift (Fig. 1d) that reduces the ion energy after the injection from above the potential height of the ion mirrors down to the trapping energy. In analogy, the ion energy is raised back up for ejection.

Typical energies of the (singly charged) stored ions are around $2 \mathrm{keV}$. For ion separation the mass analyzer is followed by a Bradbury-Nielsen ion gate [11,38,39]. Mass resolving powers (FWHM) of 100,000 and 200,000 are achieved at trapping times of $12 \mathrm{~ms}$ and $30 \mathrm{ms,}$ respectively $[7,11]$. These are short compared with typical excitation times of the order of hundreds of milliseconds for selecting isobars in the preparation Penning trap. With respect to mass measurements the reduction of time consumption is even higher because the cyclotron resonance (top spectrum in Fig. 2) is measured by scanning the excitation frequency with durations of typically a second for each frequency point. Thus, a single scan with 41 frequency points (top inset in Fig. 2) lasts about a minute. In contrast, MR-ToF mass spectrometry is a non-scanning method, where all ions observed contribute fully to the time-of-flight and thus to the mass determination. On the other hand, the Penning-trap mass measurements achieve higher resolving powers and mass accuracies.

The lower spectrum of Fig. 2 is a logarithmic plot of the MR-ToF ion signal as a function of flight time for an isobaric mixture at mass number 149. It was taken in the context of an extended study of the accuracy of the MR-ToF measurements whose data is currently under evaluation. The ToF-ICR method requires a reference measurement for magnetic field calibration. In contrast, the MR-ToF method uses the simultaneously monitored flight times of other ion species. In other words: The ToF-ICR contamination ions become MR-ToF calibration ions. On the other hand, ToF-ICR measurements can provide an identification of MR-ToF signals in cases where the accuracy of the MR-ToF values is not sufficient. Thus, both methods are complementary and mutually beneficial.

\subsection{Applications of ISOLTRAP's MR-ToF mass analyzer}

Initially, the MR-ToF mass separator was intended to support and facilitate the Penningtrap mass measurements by supplying isobarically purified ion-of-interest samples with halflives on the order of a second or below. This has been demonstrated with the first mass measurement of the short-lived species ${ }^{82} \mathrm{Zn}$ [23], an important ingredient for calculations of the composition of neutron-star crusts. Furthermore, a Penning-trap stacking technique has been implemented that increases the number of trapped purified ions without increasing the space charge in the MR-ToF system. Thus, the measurement time for highly contaminated ion beams with half-lives around a second or above can be reduced by about an order of magnitude [40]. 
Furthermore, the MR-ToF device was employed on-line for the first mass measurements of the very neutron-rich calcium isotopes ${ }^{53} \mathrm{Ca}$ and ${ }^{54} \mathrm{Ca}$. In particular for ${ }^{54} \mathrm{Ca}$, with an ISOLDE ion yield of only 10 ions/s and a half-life of 86(3) ms [41], the mass was determined with a statistical uncertainty below one ppm. In this case, the isobaric chromium "contaminants" of the ISOLDE ion beam were used as reference masses. The successful MR-ToF mass determinations underlined the magicity of the neutron number $N=32$ in this region of the nuclear chart [12].

The MR-ToF device has also shown to be very beneficial for target and ion-source developments at the ISOLDE facility [7,16,24]. In contrast to the other methods available, i.e. Faraday cups for picoampere ion-beams or beam identification by use of decay stations, the MR-ToF mass analyzer opened the possibility to acquire qualitative and quantitative information from highly resolved isobaric spectra, independent of half-life or decay mode, with a dynamic range of several orders of magnitude. Thus, it closed the gap between high-intensity beams of long-lived nuclides and low-intensity beams of very short-lived nuclides.

Finding efficient excitation schemes for resonance ionization at ISOLDE's laser ion source can also be supported by MR-ToF mass spectrometry [15,24]. In fact, this application is closely related to studies of isotope shifts and hyperfine structures of short-lived nuclides, which leads directly back to the investigation of the fundamental properties of these exotic species. In short, there is more than masses for the MR-ToF mass analyzer of ISOLTRAP.

\section{MR-ToF studies at Greifswald}

Another MR-ToF device was recently built at Greifswald [42] for the advanced physicslaboratory course. In addition, it is used to study ion-ion interactions and space-charge effects, which become important at high ion densities, for example when highly contaminated beams are to be handled. In the following, preliminary results of recent measurements are described.

Already more than a decade ago "self-bunching" has been observed [43-45], i.e. the phenomenon that in spite of their repulsing Coulomb interaction the ions do not spread out but on the contrary - form rather short pulses. Similar to the "peak coalescence" effect observed in Fourier-Transform Ion Cyclotron Resonance (FT-ICR) mass spectrometry [46-48] "selfbunching" can lead to a merging of signals of different ion species [49].
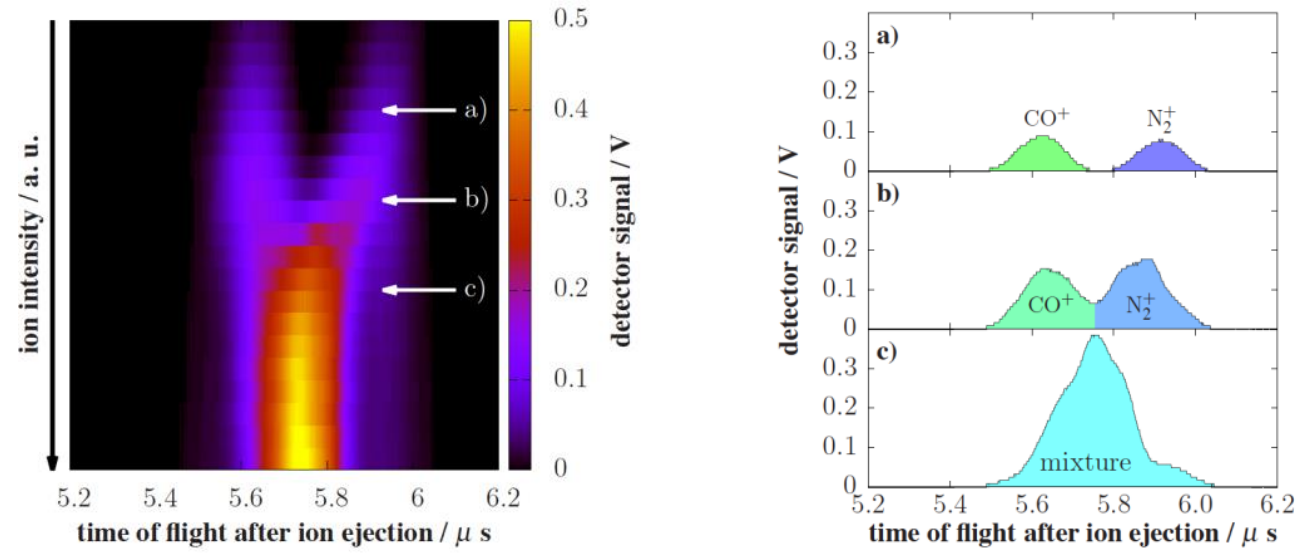

Fig. 3: Peak-coalescence of $\mathrm{CO}^{+}$and $\mathrm{N}_{2}^{+}$signals monitored as a function of the ion intensity. The ToF spectra (right) correspond to the cases marked by the arrows (left). 
This phenomenon was probed experimentally for the case of the molecular isobar doublet $\mathrm{CO}^{+} / \mathrm{N}_{2}{ }^{+}$, at mass number $A=28$, which requires a mass resolving power of $R_{\mathrm{FWHM}} \approx 2,800$ for equal ion intensities. For the present measurements, the ions are produced by electron-impact ionization in a linear Paul trap and forwarded with a mean kinetic energy of $2 \mathrm{keV}$ towards the MR-ToF device. There, they are captured by means of the in-trap lift technique and stored for $1.6 \mathrm{~ms}$ at a kinetic energy of $1 \mathrm{keV}$. The number of ions can be adjusted by modifying the trapping conditions of the Paul trap, e.g. by change of the rf-amplitude of the guiding field. After ejection from the MR-ToF device, the ions are detected by a micro-channel plate (MCP) detector. Its signal is fed to an oscilloscope for data acquisition.

In Fig. 3 an example of the evolution of ToF spectra with increasing number of ions is shown (contour plot on the left, from top to bottom): For a few hundred ions (case a), the peaks corresponding to $\mathrm{CO}^{+}$(left) and $\mathrm{N}_{2}^{+}$(right) are well separated. With increasing number of ions their ToF distance decreases (case b) until the peaks are no longer separated, corresponding to some thousand ions in the trap (case c). As the MCP detection signals are not necessarily linear for these dense ion bunches, the ion numbers are only rough estimates.

In addition, the ions' kinetic energy distributions were investigated by monitoring the ion intensity as a function of a blocking voltage applied to deceleration grids in front of the MCP detector. The ion intensity was chosen in a way that $\mathrm{CO}^{+}$and $\mathrm{N}_{2}{ }^{+}$could still be separated in the spectra, but space-charge effects were already present. The ToF spectra in Fig. 4 (left) for different values of the blocking voltage indicate a different mean kinetic energy (while the initial kinetic energies can be assumed to be equal for both species as they are created simultaneously in the Paul-trap ion source). The area of the ToF signals for each species as a function of the blocking voltage and their first derivative is shown in Fig. 4 (right).
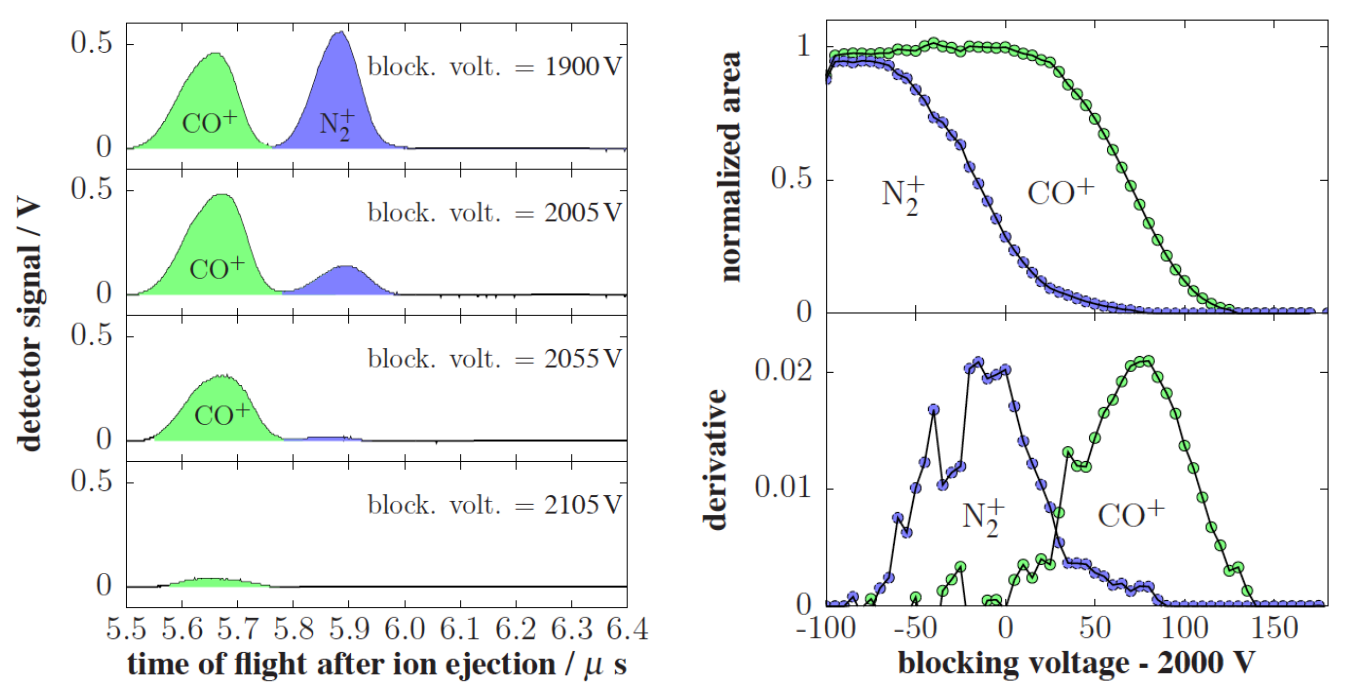

Fig. 4: Experimental MR-ToF spectra from the Greifswald MR-ToF setup for several blocking voltages (left) and integrated and normalized areas of the ToF peaks and their derivatives as a function of the blocking voltage (right). (Note that due to different detector conditions the signal heights are not the same as those of Fig. 3.) 
Apparently, the difference is due to an energy transfer during the ion storage in the MRToF. The energy separation corresponds to ten percent of the total initial mean kinetic energy in the MR-ToF MS. This feature could be of interest for ion separation by energy-dependent iontransfer systems, i.e. electrostatic devices, without switching of ion-beam gates. However, the energy-separation is restricted to specific conditions with respect to absolute intensities and to abundance ratios as well as particular MR-ToF settings. If the ion intensity in the MR-ToF device is reduced the effect decreases, and in case of only a few ions it could not be measured. Further investigations will be necessary to understand and make use of these effects.

\section{Conclusion and Outlook}

ISOLTRAP's MR-ToF mass analyzer has demonstrated for the first time on-line mass separation of isobaric nuclides as well as precision mass measurements of very short-lived species with low ion yields, which resulted in valuable input for studies of the crustal composition of neutron stars and for nuclear structure at extreme neutron-to-proton asymmetries, respectively. In addition, proof-of-principle experiments for several applications beyond precision mass determination have been performed.

Since the method is still relatively new, there are further aspects to be studied in detail. As an example, preliminary results of recent investigations in a second MR-ToF device indicate that space-charge effects have to be considered as they may limit the performance with respect to resolving different ion species and - due to the corresponding time-of-flight shifts - also with respect to the achievable mass accuracy. These effects are currently under further investigation.

\section{Acknowledgments}

This work was supported by the German Ministry for Education and Research (BMBF) under the contracts 05P09HGFNE, 05P12HGCI1, 06GF9101I, and 05P12HGFNE. We thank the ISOLDE Collaboration as well as the ISOLDE technical group for their assistance.

\section{References}

[1] K. Blaum, Phys. Rep. 425, 1 (2006).

[2] L. Schweikhard and G. Bollen (Eds.), Ultra-accurate mass determination and related topics, Special issue of Int. J. Mass Spectrom., Vol. 251 (2-3), Elsevier, Amsterdam, 2006.

[3] D. Lunney et al., Rev. Mod. Phys. 75, 1021 (2003).

[4] F. Bosch, Yu. A. Litvinov, Int. J. Mass Spectrom. 349, 151 (2013).

[5] G. Bollen et al., Hyperfine Interactions 38, 793 (1987).

[6] G. Bollen et al., Phys. Rev. C 46, R2140 (1992).

[7] R.N. Wolf et al., Int. J. Mass Spectrom. 349-350, 123 (2013).

[8] G. Savard et al., Phys. Lett. A 158, 247 (1991).

[9] L. Schweikhard et al., Eur. J. Mass Spectrom. 11, 457 (2005). 
[10] H. Wollnik, M. Przewloka, Int. J. Mass Spectrom. Ion Processes 96, 267 (1990).

[11] R.N. Wolf et al., Nucl. Instrum. Meth. A 686, 82 (2012).

[12] F. Wienholtz et al., Nature 498, 346 (2013).

[13] Y. Ito et al., Phys. Rev. C 88, 011306R (2013).

[14] W.R. Plaß et al., Nucl. Instrum. Meth. B 317, 457 (2013).

[15] B.A. Marsh et al., Nucl. Instrum. Meth. B 317, 550 (2013).

[16] A. Gottberg et al., Nucl. Instrum. Meth. B 336, 143 (2014).

[17] W.C. Wiley, I. H. McLaren, Rev. Sci. Instrum. 26, 1150 (1955).

[18] B.A. Mamyrin et al., Soviet Physics Jetp-Ussr 64, 82 (1973).

[19] D. Zajfman et al., Phys. Rev. A 55, R1577 (1997).

[20] W.H. Benner, Analytical Chemistry 69, 4162 (1997).

[21] M. Lange et al., Rev. Sci. Instrum. 81, 055105 (2010).

[22] A. Piechaczek et al., Nucl. Instr. and Meth. B 266, 4510 (2008).

[23] R.N. Wolf et al., Phys. Rev. Lett. 110, 041101 (2013).

[24] S. Kreim et al., Nucl. Instrum. Meth. B 317, 492 (2013).

[25] H. Stolzenberg et al., Phys. Rev. Lett. 65, 3104 (1990).

[26] G. Bollen et al., Nucl. Instr. Meth. A 368, 675 (1996).

[27] G. Mukherjee et al., Eur. Phys. J. A 35, 1 (2008).

[28] E. Kugler, Hyperfine Interactions 129, 23 (2000).

[29] F. Herfurth et al., Nucl. Instrum. Meth. A 469, 254 (2001).

[30] L.S. Brown, G. Gabrielse, Rev. Mod. Phys. 58, 233 (1986).

[31] H. Raimbault-Hartmann et al., Nucl. Instrum. Meth. B 126, 378 (1997).

[32] G. Bollen et al., J. Appl. Phys. 68, 4355 (1990).

[33] M. König et al., Int. J. Mass Spectrom. Ion Process. 142, 95 (1995).

[34] A. Kellerbauer et al., Eur. Phys. J. D 22, 53 (2003).

[35] R.N. Wolf et al., Hyperfine Interactions 199, 114 (2011).

[36] M. Dahan et al., Rev. Sci. Instrum. 69, 76 (1998).

[37] R.N. Wolf et al., Int. J. Mass Spectrom. 313, 8 (2012).

[38] N.E. Bradbury, R.A. Nielsen, Phys. Rev. 49, 388 (1936).

[39] W.R. Plaß et al., Nucl. Instr. and Meth. B 266, 4560 (2008). 
[40] M. Rosenbusch et al., Appl. Phys. B 114, 147 (2014).

[41] G. Audi et al., Chinese Phys. C 36, 1157 (2012).

[42] M. Rosenbusch et al., AIP Conf. Proc. 1521, 53 (2013).

[43] H.B. Pedersen, et al., Phys. Rev. Lett. 87, 055001 (2001).

[44] H.B. Pedersen et al., Phys. Rev. A 65, 042704 (2002).

[45] D. Strasser et al., Phys. Rev. Lett. 89, 283204 (2002).

[46] Y. Naito, M. Inoue, J. Mass Spectrom. Soc. Jpn. 42, 1 (1994).

[47] I.A. Boldin, E. N Nikolaev, Rapid Commun. Mass Spectrom. 23, 3213 (2009).

[48] G. Vladimirov et al., J. Am. Soc. Mass Spectrom. 23, 375 (2012).

[49] P.A. Bolotskikh et al., Nucl. Instr. Meth. A 645, 146 (2011). 